\title{
Inhibition of Plasmodium falciparum Hsp90 Contributes to the Antimalarial Activities of Aminoalcohol-carbazoles
}

\author{
Tai Wang, ${ }^{\dagger, \|}$ Pascal Mäser, ${ }^{\ddagger}, \S$ and Didier Picard ${ }^{* \dagger}$
}

†Département de Biologie Cellulaire, Université de Genève, Sciences III, 30 Quai Ernest-Ansermet, CH-1211 Genève 4, Switzerland

${ }^{\ddagger}$ Swiss Tropical and Public Health Institute, Socinstrasse 57, CH-4051 Basel, Switzerland

${ }^{\S}$ University of Basel, Petersplatz 1, CH-4001 Basel, Switzerland

\section{Supporting Information}

ABSTRACT: Malaria caused by the protozoan parasite Plasmodium falciparum ( $P f$ ) remains a major public health problem throughout the developing world. One molecular target that should receive more attention is the molecular chaperone Hsp90. It is essential and highly conserved in all eukaryotes, including in protozoan parasites. We have identified an aminoalcohol carbazole (N-CBZ) as a PfHsp90-selective inhibitor by virtually docking a large set of antimalarial compounds, previously found in a phenotypic screen, into a PfHsp90-specific pocket. By correlating the ability of 30 additional N-CBZ derivatives to bind directly to PfHsp90 with their Pf-inhibitory activity, we found that these types of compounds are more likely to inhibit Pf growth if they bind PfHsp90. For plausible targets such as PfHsp90, our workflow may help identifying the molecular target for compounds found by screening large chemical libraries for a desired biological effect and, conversely, ensuring biological effectiveness for compounds affecting a particular target.

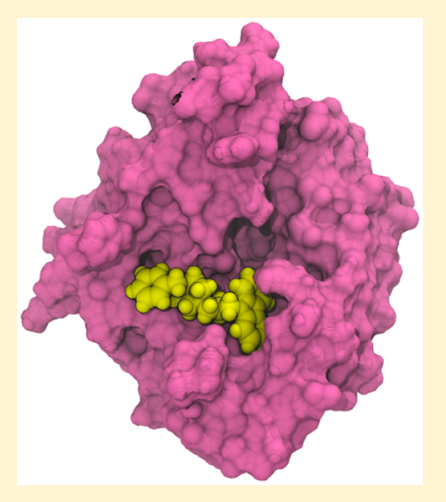

\section{INTRODUCTION}

Malaria is caused by infection with protozoan parasites such as Plasmodium falciparum $(P f)$. It remains a deadly disease that affects 104 countries, causing a global death toll of more than 600000 and putting 3.4 billion people in danger. Effective antimalarials are therefore indispensable tools for public health in many countries. Unfortunately, current antimalarial drugs are rapidly losing their effectiveness because of arising parasite resistance. This calls for the discovery and development of novel agents with high effectiveness and selectivity. ${ }^{1,2}$ More and more of these recent efforts have used combinations of computational and experimental strategies to target a variety of cellular processes of $P f$. . $^{3,4}$

Hsp90 is a highly abundant and conserved cytosolic molecular chaperone, which is essential to maintain protein homeostasis in eukaryotes. ${ }^{5,6}$ This also applies to protozoan parasites: Numerous pharmacological studies with generic Hsp90 inhibitors have established the parasite Hsp90 as a molecular target against malaria; inhibition of Hsp90 of Pf (PfHsp90) not only blocks the lifecycle of the parasite at the red blood cell stage but also leads to parasitemia clearing in a mouse model. $^{7-15}$ Another relevant aspect of Hsp90 biology is that Hsp90 supports the development of drug resistance both in $P f$ and pathogenic fungi, and Hsp90 inhibitors therefore not only interfere with a multitude of essential cellular functions but also many resistance mechanisms. ${ }^{15-18}$ Hence, inhibitors targeting Hsp90 hold great promise as next-generation antimalarial drugs, notably if they can be developed to be selective for Hsp90 of the parasite over that of the human host. $^{19,20}$ The problem is that $\mathrm{PfHsp} 90$ has a very high sequence homology with its human orthologues, which has impeded the development of the known Hsp90 inhibitors into realistic treatment options for malaria. To overcome this issue, we recently reported a novel drug design strategy which takes advantage of a Pf-specific drug binding pocket in PfHsp90. This allowed us to discover a 7 -azaindole as a species-selective PfHsp90 inhibitor. ${ }^{20}$ Despite binding PfHsp90 in a speciesselective fashion and inhibiting Pf proliferation in culture, this 7-azaindole still requires substantial optimization of binding affinity, antimalarial potency, and presumably its pharmacological profile.

High-throughput phenotypic screens of large compound libraries have yielded many new antimalarial candidates in the past few years. ${ }^{21-26}$ These compounds have high potency and selectivity against Pf and excellent pharmacological profiles. Phenotypic screens are undoubtedly a powerful approach to discover novel lead compounds. Their big advantage is that from the outset it is clear that hits have the desired effect in a given biological assay system. One of the main disadvantages, however, is that they do not automatically reveal the molecular target(s) whose inhibition or activation underlie the biological effects. Without knowing the molecular target and mechanism of action, any further rational drug development is severely compromised. This dilemma prompted us to consider combining opposite drug discovery strategies. Here, we propose a reversed drug discovery approach that aims at

Received: April 17, 2016

Published: June 17, 2016 


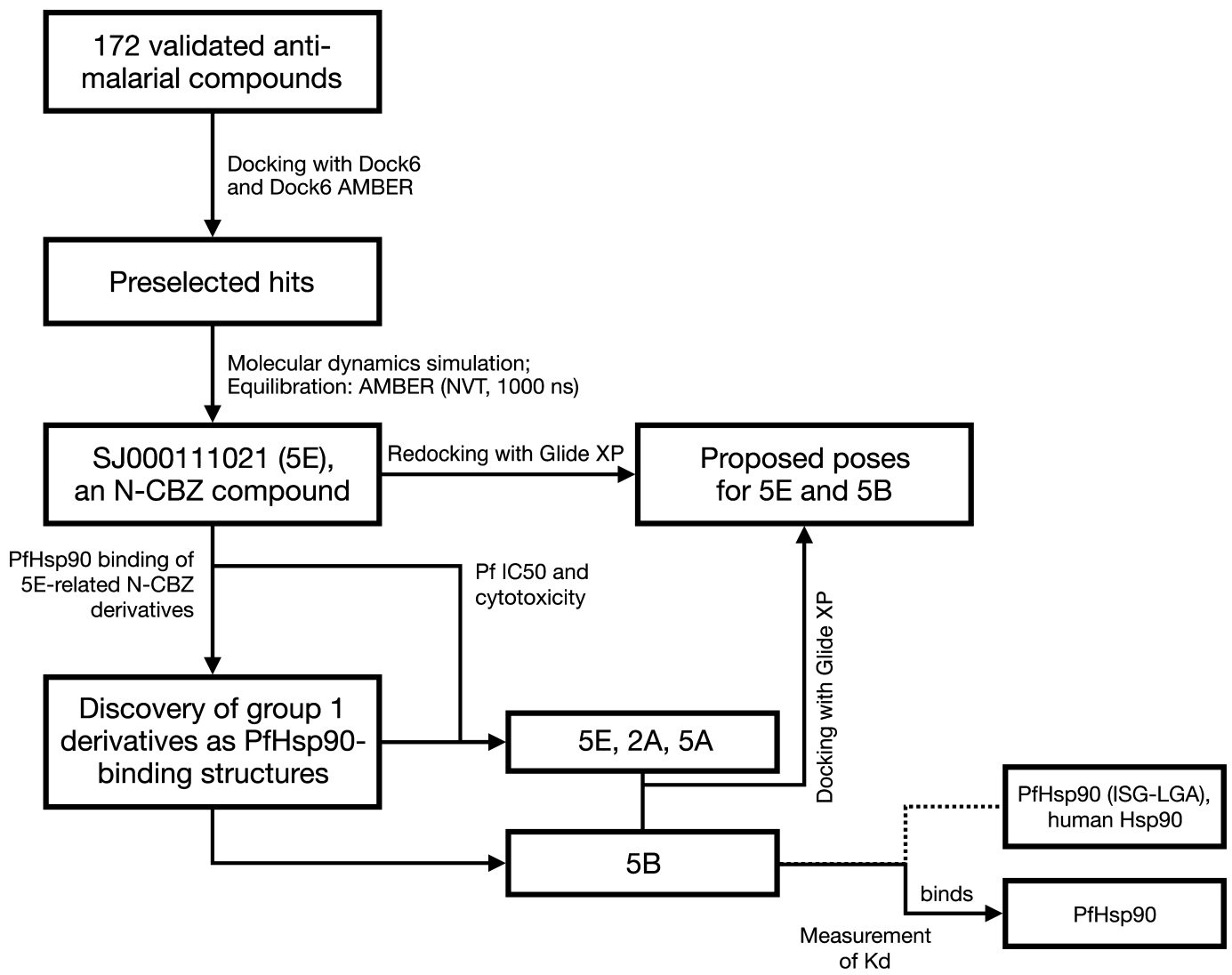

Figure 1. Workflow for identifying and characterizing antimalarial compounds that target PfHsp90.

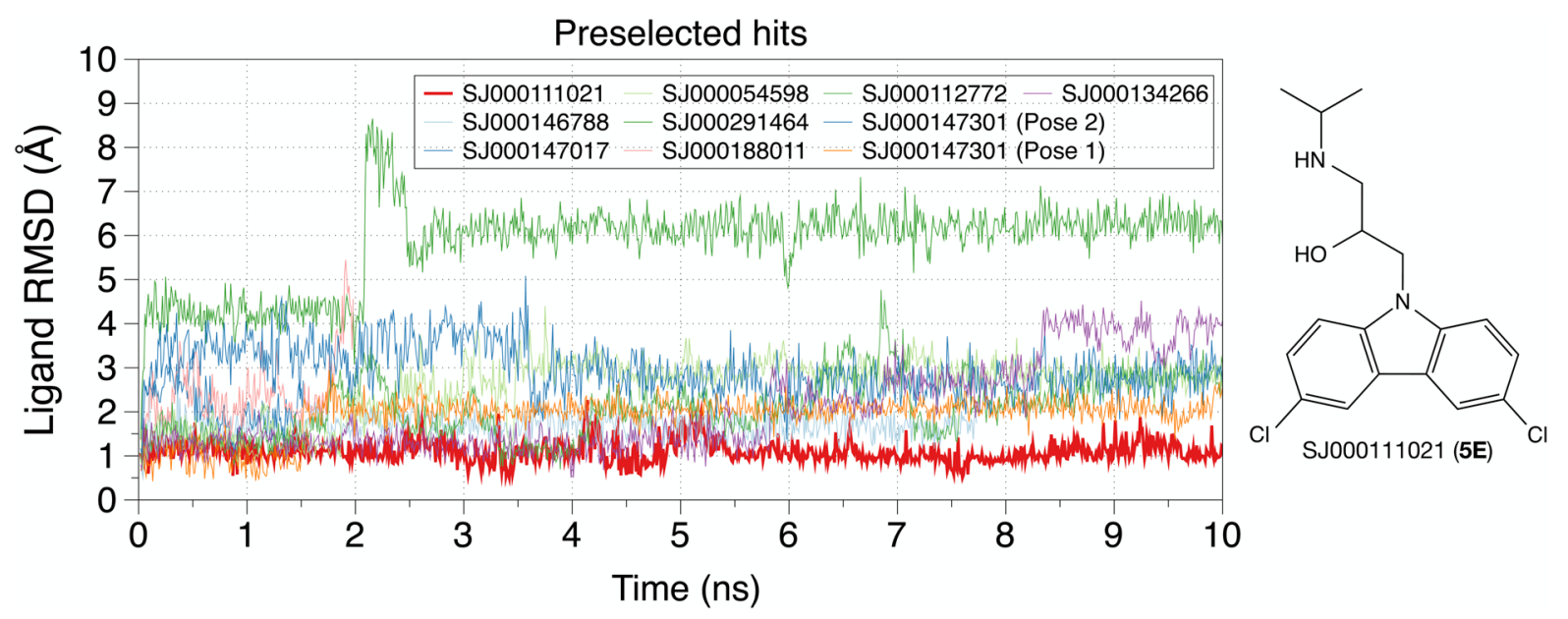

Figure 2. MD simulation of 10 hits preselected by docking to PfHsp90 from 172 antimalarial compounds. The nomenclature of the compounds is the original one from the publication by Guy and colleagues. ${ }^{21}$ The structure of compound SJ000111021 (referred to here as 5E), which displays a

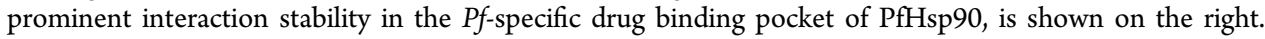

discovering PfHsp90 inhibitors among libraries of potent but poorly characterized antimalarial compounds.

\section{RESULTS}

We hypothesized that Hsp90-targeting compounds may be represented in a library of antimalarial compounds of sufficient chemical diversity and designed a strategy to identify and to characterize them (Figure 1). We took all 172 validated antimalarial compounds from a previously published large phenotypic screen $^{21}$ and docked them in silico within the Pfspecific drug binding pocket of PfHsp90. To validate and refine the in silico docking results, we performed molecular dynamics (MD) simulations using the original docking pose of the 10 top-ranked hits (Supporting Information, Table S1) in the Pfspecific drug binding pocket of PfHsp90 and examined their interaction and stability along the simulation (Figure 2). While most of the compounds became unstable and eventually dissociated, we noticed that compound SJ000111021 (hereafter referred to as compound $5 \mathrm{E}$ ) retained its original pose and demonstrated a high stability inside the $P$-specific pocket. $5 \mathrm{E}$ is a compound based on aminoalcohol-carbazole (N-CBZ). We cross-validated this result with Glide, another docking software, 
and found a predicted top pose which was very similar to that obtained with the original docking and MD simulations (Supporting Information, Table S2). The predicted pose has the tricyclic CBZ ring, together with a chloride at its 3-position, anchored in the Pf-specific pocket delimited by THR101, PHE104, and the glycine-rich hinge loop between GLY118 and GLY123 (Figure 3). Both the hydroxyl group and the

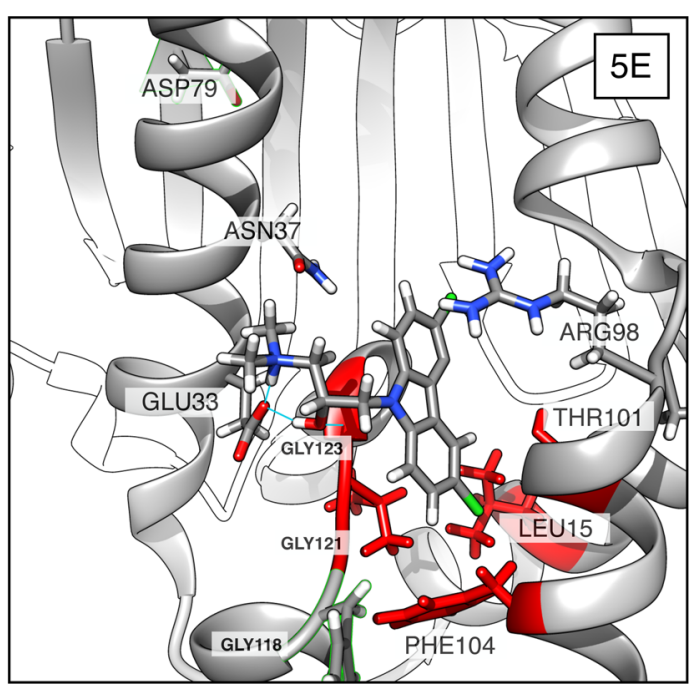

Figure 3. Proposed docking pose of compound $\mathbf{5 E}$ in the Pf-specific drug binding pocket of $\mathrm{PfHsp} 90$ (labeled in red).

secondary amine on the $N$-alkyl branch of $5 \mathrm{E}$ are predicted to form $\mathrm{H}$-bonds with the deprotonated carboxyl group on the side chain of GLU33 of PfHsp90. This model suggests that the chloro-CBZ core of $\mathbf{5 E}$ provides the main van der Waals interaction with the surrounding residues of the $P f$-specific pocket of PfHsp90 while the $N$-alkyl chain essentially contributes an electrostatic interaction. These in silico studies indicated that N-CBZ derivatives such as compound 5E may target PfHsp90.

For our next experimental steps, we acquired $30 \mathrm{~N}-\mathrm{CBZ}$ derivatives and subjected them to a structure-activity relationship (SAR) analysis. To determine whether these compounds indeed bind PfHsp90, we performed microscale thermophoresis experiments with recombinant $\mathrm{PfH}$ sp90. This technology exploits the ligand-induced changes in the molecular movements of the fluorescently labeled protein in a temperature gradient, which are a measure of its binding affinity. We determined the thermophoresis shifts of all compounds at a fixed concentration of $50 \mu \mathrm{M}$ (Table 1 and Supporting Information, Tables S2 and S3). These analyses revealed that many N-CBZ derivatives directly bind to PfHsp90 and confirmed the in silico prediction with compound 5E. Because this SAR analysis indicated marked differences between compounds, we sought to identify the molecular determinants of binding. This analysis was performed with ChemmineR, ${ }^{27}$ a basic tool for quantitative SAR (QSAR). We clustered the compounds by chemical similarity and displayed the hierarchical tree along with the binding results represented as a heatmap (Figure 4, Table 1, and Supporting Information, Table S3). This clearly segregates the compounds into two groups where those of group 1, which are characterized by a piperazine group at the end of the $\mathrm{N}$-alkyl group, appear more likely to bind PfHsp90. We selected compound $\mathbf{5 B}$ as a representative of group 1 and determined its binding affinity for
Table 1. Structures, Biochemical, and Biological Activities of Tested Compounds ${ }^{a}$

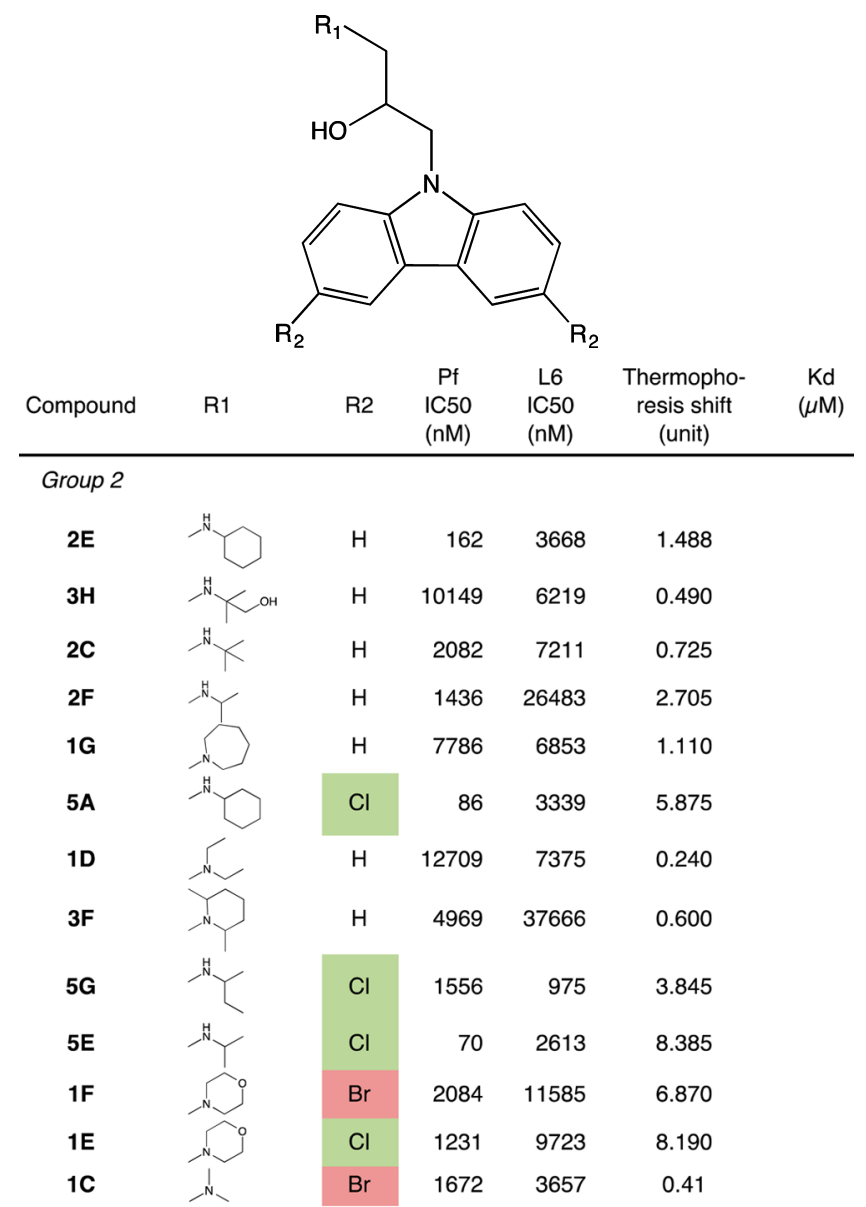

Group 1
Cl

$a_{*}$ This compound is identical to compound 14a in ref 28 .

PfHsp90 and human Hsp90 $\beta$ by microscale thermophoresis (Figure 5 and Table 1). We found that 5B binds PfHsp90 with a $K_{\mathrm{d}}$ of $28.1 \mu \mathrm{M}$ but does not bind human Hsp90 $\beta$ at all. The closely related chemical 3B binds PfHsp90 with a $K_{\mathrm{d}}$ of 25.6 $\mu \mathrm{M}$ (Supporting Information, Figure S1). The pan-Hsp90 inhibitor geldanamycin binds Hsp90 from both species with similarly high affinities.

Considering our modeling and in vitro binding experiments, it seemed likely that $\mathbf{5 B}$ binds in the Pf-specific pocket of PfHsp90. To provide experimental evidence for this hypothesis, we proceeded with binding studies using the PfHsp90 triple mutant ISG-LGA (I108L, S111G, G112A), in which the Pfspecific pocket is closed and resembles the configuration of human Hsp90 $\alpha$. As expected, the PfHsp90 ISG-LGA changes prevent the binding of $\mathbf{5 B}$ (Figure 5). In contrast, 


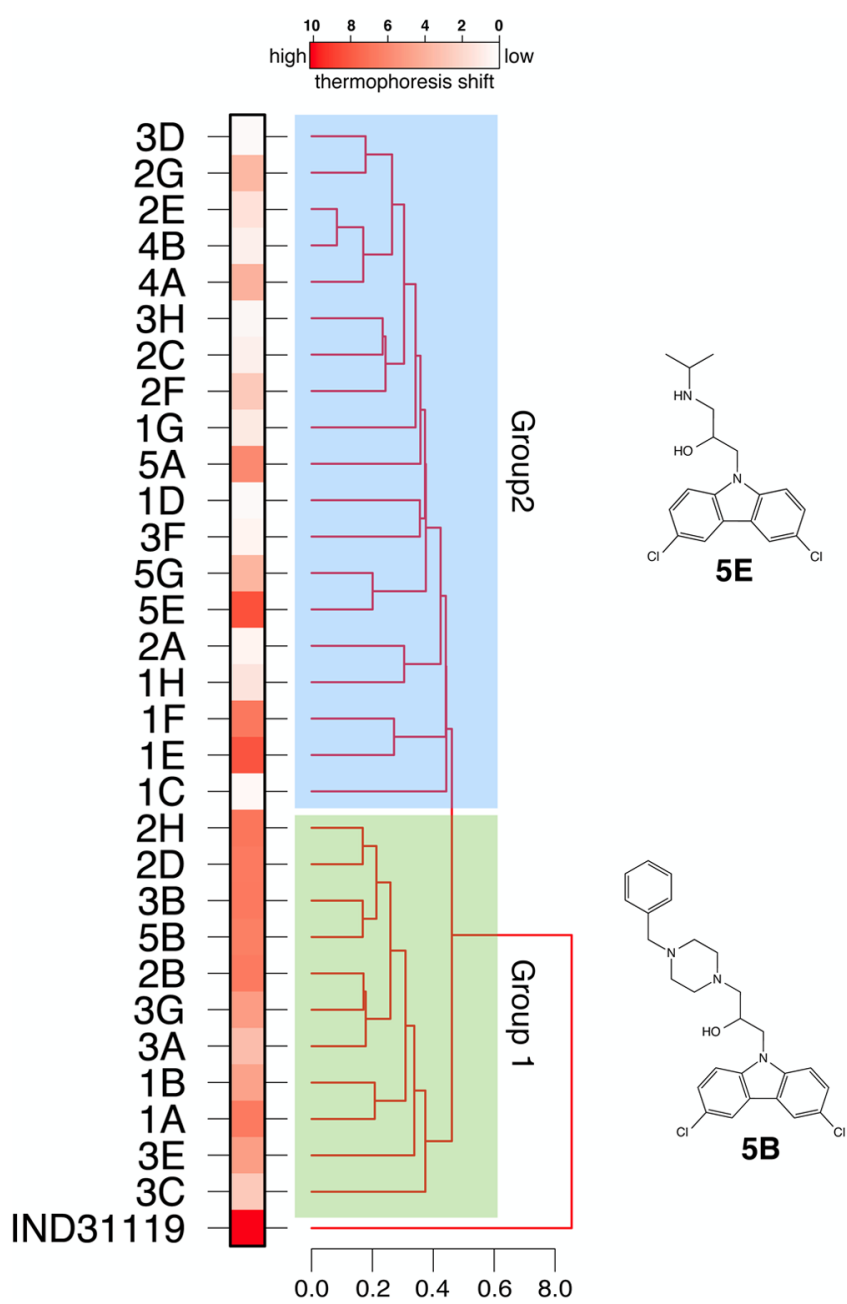

Figure 4. Scheme juxtaposing ligand binding data and clustering by chemical similarity. Binding of N-CBZ derivatives to PfHsp90 at 50 $\mu \mathrm{M}$ was determined by microscale thermophoresis, and the results are represented as a heat map where red corresponds to a large shift and thus stronger binding (higher affinity). The chemicals are clustered based on chemical similarity. The PfHsp90-selective 7-azaindole compound IND31119 ${ }^{20}$ is included as an outgroup and for comparison.

geldanamycin, which does not use the $P f$-specific pocket for binding, binds the triple mutant with similar affinity. These findings strongly support the conclusion that compound 5B binds PfHsp90 in a species-selective fashion because of its engagement of the Pf-specific pocket.

To provide further computational support for the direct binding of $\mathbf{5 B}$ in the Pf-specific drug binding pocket of PfHsp90, we performed docking studies using Glide (Supporting Information, Table S2 ). We found that two prominent poses can be predicted and that in both cases the chloro-CBZ core occupies the Pf-specific pocket (Figure 6). In predicted pose 1 , the CBZ core adopts a "flipped-in" conformation with its $N$-alkyl branch reaching into the Hsp90 ATP binding pocket surrounded by ASN37, ASP79, VAL122, GLY123, THR171, and ILE173. In contrast, predicted pose 2 consists of a "flippedout" CBZ conformation, leaving the Hsp90 ATP and standard drug binding pocket vacant. In this case, the $N$-alkyl branch protrudes over the glycine-riched loop (GLY118 to GLY123) at the entrance of the Hsp90 ATP binding pocket. The predicted pose 2 for $\mathbf{5 B}$ is very similar to the favored docking pose of $\mathbf{5 E}$ (compare Figures 3 and 6), and for both compounds, there are H-bonds between the carboxyl group of GLU33 and the $\mathrm{N}$ alkyl group.

At this point, we had established that some N-CBZ compounds bind PfHsp90 directly, albeit with modest affinity, and can be modeled into the Pf-specific pocket. We selected a representative set of $\mathrm{N}-\mathrm{CBZ}$ derivatives from groups 1 and 2 and tested their ability to inhibit the proliferation of $P f$ in culture (Table 1). We found remarkably potent candidates with $\mathrm{IC}_{50} \mathrm{~s}$ of less than $200 \mathrm{nM}$ in both groups, including the two molecules (5E and 5B) that we had investigated in more detail. These also displayed only moderate cytotoxicity in rat L6 cells, which are a valid in vivo model for human Hsp90 considering that human and rodent Hsp90s are 99\% identical. In L6 cells, the whole panel of compounds gave a wide range of toxic concentrations (Table 1); this is consistent with the notions that some PfHsp90-selective inhibitors may still bind mammalian Hsp90 to some extent, albeit with poor affinity and thus low occupancy ${ }^{20}$ and/or target a variety of other molecules. Interestingly, Guy and colleagues had reported considerably higher $\mathrm{IC}_{50}$ values against $\mathrm{Pf}$ for compound $\mathbf{5 E}$, ranging from 0.48 to $1.8 \mu \mathrm{M}$ in $P f$ strains $\mathrm{K} 1$ and 3D7, respectively, ${ }^{21}$ compared to $82 \mathrm{nM}$ in our own tests with the Pf strain NF54. The $\mathrm{IC}_{50}$ s reported by Routier and colleagues in their characterization of $\mathrm{N}-\mathrm{CBZ}$ antimalarial activities with $\mathrm{Pf}$ strain $\mathrm{K}^{28}$ are far more consistent with those that we have determined. Apart from strain differences, additional experimental differences must explain these apparent numerical discrepancies. In any case, all of these data then allowed us to explore whether there is a correlation between PfHsp90 binding and inhibition of $P f$ in culture. As can be seen in Figure 7, compounds with weak PfHsp90 binding (with a thermophoresis shift $<5$ ) are much less likely to have an $\mathrm{IC}_{50}$ lower than $1 \mu \mathrm{M}$, with the exception of compound $2 \mathrm{E}$. In contrast, compounds with stronger binding to $\mathrm{PfHsp90}$ (with a thermophoresis shift $>5$ ) tend to have an $\mathrm{IC}_{50}$ lower than 100 $\mu \mathrm{M}$, as exemplified most prominently by compounds $\mathbf{5 B}, \mathbf{2 D}$, $5 \mathrm{E}$, and $5 \mathrm{~A}$.

\section{DISCUSSION AND CONCLUSIONS}

Phenotypic screens for active compounds can yield interesting candidates but may not provide any insights into their molecular targets. We developed a workflow (Figure 1) to deorphanize active compounds in a context where the molecular chaperone Hsp90 was a plausible target. We discovered that a library of previously identified antimalarial compounds ${ }^{21}$ contained an N-CBZ whose molecular target is, or at least includes, PfHsp90.

CBZ derivatives have been known for their antimalarial potency for some time. ${ }^{29,30} \mathrm{~N}$-Substituted CBZ derivatives were first identified as potent antimalarials with a phenotypic screen against the chloroquine-resistant Pf strain K1. ${ }^{26}$ More recently, an extensive SAR analysis of the derivatives bearing modifications at the 3-and/or 6-positions and the $\mathrm{N}$-alkyl of the CBZ core led to the identification of several N-CBZ derivatives with promising antimalarial and pharmacokinetic characteristics. $^{28}$ The N-CBZ pharmacophore came up in our own pipeline through an entirely different and unbiased approach when we discovered compound $5 \mathrm{E}$, one of 172 , in the antimalarial library of compounds found in a phenotypic screen with almost 400000 compounds. ${ }^{21}$ However, neither the $\mathrm{N}$-CBZ-targeted ${ }^{28}$ nor the high-throughput screen ${ }^{21}$ provided any clues as to the potential drug targets and mechanisms of 
$5 B$
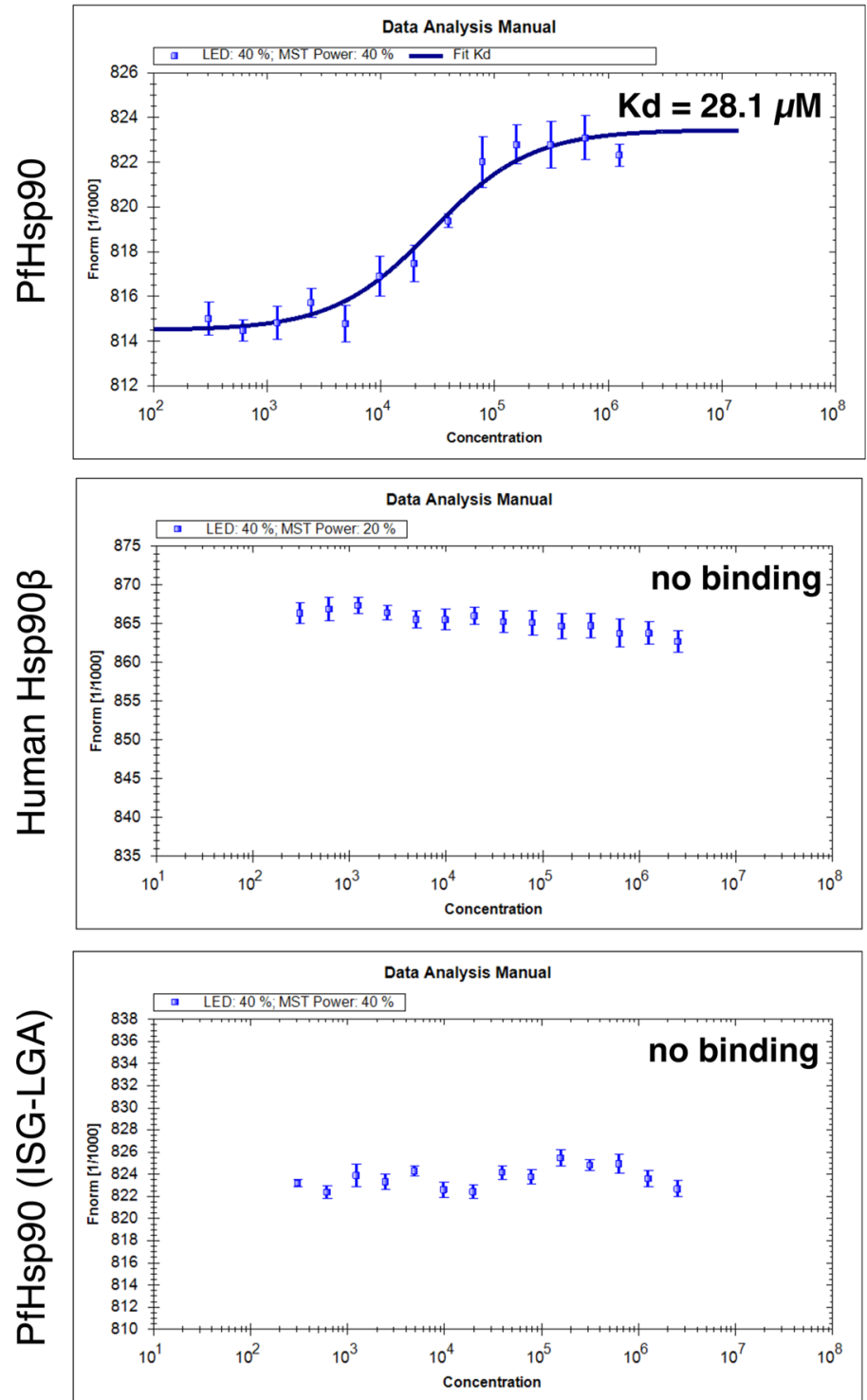

\section{Geldanamycin}
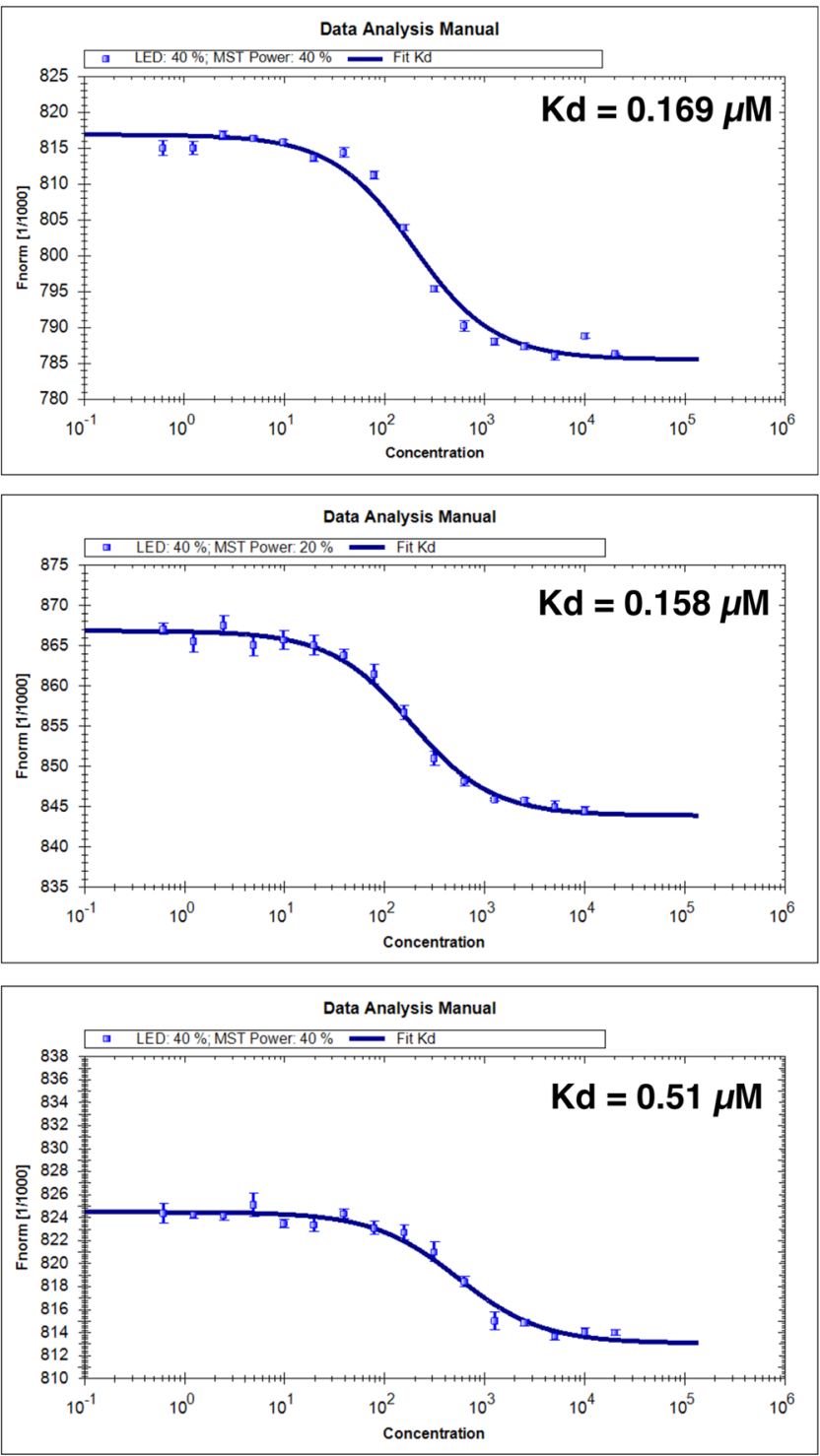

Figure 5. Microscale thermophoresis measurements of the binding affinities of compound 5B and geldanamycin for PfHsp90, human Hsp90 $\beta$, and the PfHsp90 triple mutant ISG-LGA.
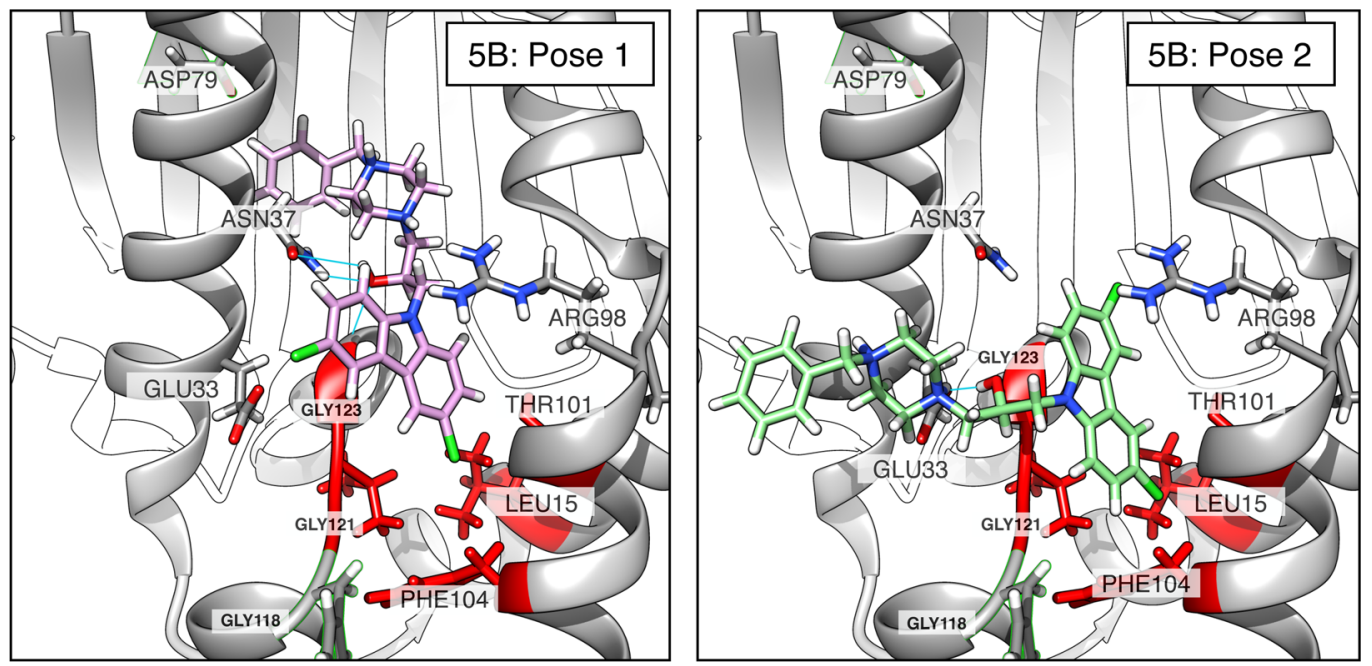

Figure 6. Proposed docking poses of compound 5B in the Pf-specific drug binding pocket of PfHsp90 (labeled in red). See text for more details. 


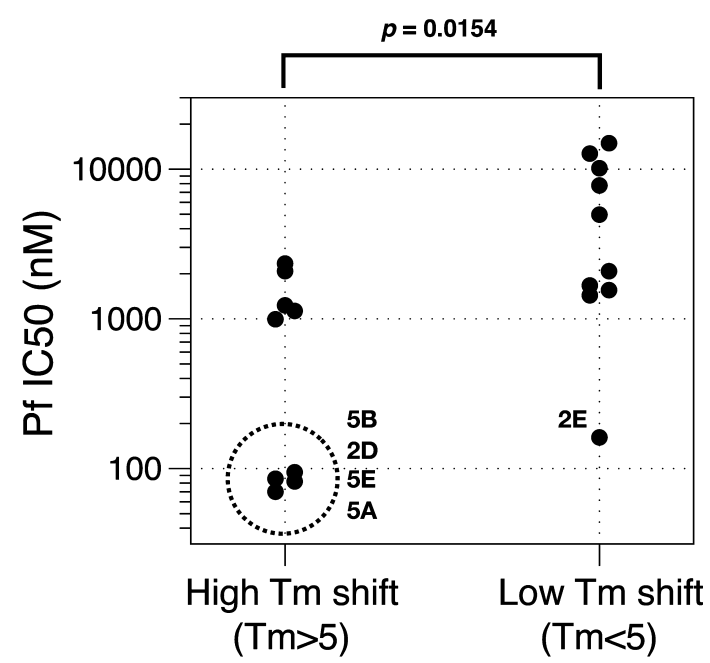

Figure 7. Structure-independent correlation between stronger PfHsp90 binding and more potent antiproliferative potency against Pf. Shifts larger or smaller than 5 in the microscale thermophoresis experiment are indicative of a higher or lower affinities, respectively. All compounds tested for activity against $P f$ are grouped according to their apparent binding affinities and placed on the $\mathrm{Y}$ axis by $\mathrm{IC}_{50}$ in the Pf proliferation assays. Student's $t$ test was performed comparing the groups with high $(n=9)$ and low $(n=10)$ thermophoresis shifts $(p$ value $=0.0154)$

action. Here, we have filled this gap by demonstrating that $\mathrm{PfH}$ sp90 is at least one target for some N-CBZ derivatives and that promising compounds may not target Hsp90s of the human host because those lack the binding pocket that they engage in PfHsp90. ${ }^{20}$ In fact, this may also explain why CBZbased compounds had not been encountered before as Hsp90 inhibitors. ${ }^{31}$ With the exception of the chemically slightly different carbazol-4-one molecules, ${ }^{32} \mathrm{CBZ}$ may not bind the canonical ATP-binding pocket of human Hsp90 (see also below).

The specificity of our workflow consisted of starting from the bold assumption that there could be Hsp90 inhibitors in a large set of antimalarial compounds and in screening them in silico and subsequently experimentally. It is this combination that allowed us to circumvent the high false-positive rates typically associated with docking results. ${ }^{33}$ First, we performed MD simulations of candidates that were top ranked in the primary docking analysis. This step was helpful to filter out the false positive candidates, which can be expected to dissociate rapidly along the MD simulation. MD simulations provided a relatively fast method with sufficient granularity for filtering compounds further before subsequent more in-depth modeling. Compound $\mathbf{5 E}$ was the sole candidate that stably interacted with the Pfspecific pocket of PfHsp90 through both van der Waals and electrostatic interactions (Figures 2 and 3). Second, because the SAR analyses of a large set of compounds can be timeconsuming and complicated, we applied a chemoinformatic analysis by which the degree of similarity of compounds can be quantitatively represented by algorithmic distances and eventually displayed by hierarchical clustering (Figure 4). Third, we directly tested PfHsp90 binding of a panel of N-CBZ derivatives based on the structure of $5 \mathrm{E}$ and found that many, although not all, of the derivatives do indeed bind PfHsp90. The hierarchical clustering based on chemical similarity revealed as a clear trend that compounds with a piperazine group at the end of the $N$-alkyl (compounds in group 1, Figure
4) are more likely to bind to PfHsp90. This preferential binding suggests of a route for further optimization. It should be mentioned that interesting hits such as $5 E$ could also be found within group 2 compounds, but elongated decorations at the $\mathrm{N}$ alkyl seem to contribute to a better binding affinity (Table 1).

Most well-characterized Hsp90 inhibitors bind to the highly conserved N-terminal ATPase pocket and, therefore, their species selectivity is weak. ${ }^{20,31,34-36}$ PfHsp90 differs from human Hsp90 by the Pf-specific pocket, a hydrophobic pocket which extends from the conserved ATP-binding pocket. $^{20,37}$ Compounds that rely on binding to the Pf-specific pocket of PfHsp90 are excellent candidates for the design of PfHsp90selective inhibitors. ${ }^{20}$ With this in mind, we performed the docking of all molecules in the Pf-specific pocket rather than the common drug-binding pocket of Hsp90. Noticing an abundance of PfHsp90 binders in group 1 compounds, we selected 5B for further in-depth analysis. We experimentally determined its binding affinity and confirmed its species selectivity. This conclusion could be further supported by showing that the PfHsp90 triple mutant ISG-LGA, which resembles human $\mathrm{Hsp} 90$ around the Pf-specific pocket, fails to bind 5B (Figure 5). Thus, it is reasonable to assume that the predicted contacts of $\mathbf{5 B}$ within the Pf-specific pocket (Figure 6) account for the species selectivity of this compound.

Our SAR analysis revealed that compounds with similar PfHsp90 binding affinities can exhibit very distinct antimalarial potency: compound $\mathbf{3 B}$ only differs from $\mathbf{5 B}$ by an extra carboxylate within the $N$-alkyl branch between the piperazine and the benzene groups, but this addition decreases the antimalarial activity by more than 1 order of magnitude (Table 1). We also found that the removal of halogens at $\mathrm{R} 2$ is detrimental to the growth inhibitory activity but does not always reduce binding to PfHsp90 (Table 1, compare 5B/2D to $2 \mathrm{~B}$ and $5 \mathrm{~A}$ to $2 \mathrm{E}$ ). Previous studies had indicated that strong inhibition of PfHsp90 alone is sufficient for effective antimalarial activity. ${ }^{7,8,12,14}$ In the case of compound 5B, a good representative of our set of active N-CBZ compounds, there is an obvious gap between its $\mathrm{PfHsp} 90$ binding affinity $\left(K_{\mathrm{d}}=24 \mu \mathrm{M}\right)$ and its potency in inhibiting $\mathrm{Pf}$ growth $\left(\mathrm{IC}_{50}=\right.$ $82 \mathrm{nM}$ ) (Table 1). This supports the speculation that N-CBZ compounds such as $\mathbf{5 B}$ are growth inhibitory for $P f$ by inhibiting both PfHsp90 and other critical target proteins that have yet to be identified. In any case, our studies support the speculation that high affinity inhibitors of PfHsp90 are more likely to be effective antimalarials (Figure 7). All of this evidence suggests that rigorous and comprehensive $\mathrm{QSAR}^{38,39}$ programs through two distinct medicinal chemistry routes might be required to optimize the PfHsp90 binding affinity while maintaining or even improving the antimalarial activity and reducing the cytotoxicity for human cells even further.

\section{EXPERIMENTAL SECTION}

Reagents. Geldanamycin was purchased from LC Laboratories and the N-CBZ compounds (as racemic mixtures) from Chembridge (San Diego, CA). All compounds were $\geq 95 \%$ pure; according to the manufacturer, the structures and $99 \%$ purity of the tested compounds were confirmed by LC/MS (see Supporting Information). All chemicals were dissolved in $100 \%$ DMSO to $10 \mathrm{mM}$ and stored at $-20{ }^{\circ} \mathrm{C}$.

Compound Library and Docking. The in silico library of the 172 antimalarial compounds was extracted from the publication by Guy and colleagues ${ }^{21}$ and prepared with the ZINC database, including all possible enantiomers (each enantiomer was treated as an individual compound). ${ }^{40}$ The protein model of PfHsp90 was obtained from the 
crystal structure of PfHsp90 (PDB code 3 K60, chain A). ${ }^{37}$ Solvent and ligand were removed. The model was minimized for 1000 steps (steepest descent) before generation of the grid and spheres in the DOCK 6 package (UCSF). ${ }^{41}$ The screening was performed using DOCK 6.3 (UCSF). All ranked candidates were rescored with the DOCK 6 AMBER package. ${ }^{42}$ The top 10 candidates were selected and subjected to further MD simulations. For in-depth predictions of binding poses, compounds $5 \mathrm{E}$ and $5 \mathrm{~B}$ with both $R$ - and $S$-enantiomers were docked in the PfHsp90 model using Glide (Schrödinger) in the XP (extra-precision) mode. ${ }^{43-45}$ The docking results from DOCK 6 and Glide and explicit descriptions of force fields used for docking are found in Supporting Information, Table S1.

Molecular Dynamics. Ligands were removed from the PfHsp90 model (PDB 3K60), and the rest of the structure, including structural water molecules, was parametrized by FF11 from AMBER11.47. A solvent box of $8 \AA$ was generated around the protein in the presence of counterions to equilibrate the charges. ${ }^{46}$ The models were first minimized in ACEMD for 250 steps, followed by $1 \mathrm{~ns}$ NVE (300 K, with harmonic constraints on the $\alpha$-carbons) and $40 \mathrm{~ns}$ NPT (with the harmonic constraints on the $\alpha$-carbons) with a cutoff of $9 \AA$, constrained H-bonds, time step of $4 \mathrm{fs}$, Langevin damping at $0.1 \mathrm{ps}-1$, and a PME frequency of $2 \mathrm{fs} .{ }^{47}$

Chemoinformatic Analyses. The chemical similarity of $30 \mathrm{~N}$ CBZ derivatives was characterized by calculating the algorithmic distance using the R package ChemmineR (https://www.r-project.org, https://www.bioconductor.org/packages/release/bioc/vignettes/ ChemmineR/inst/doc/ChemmineR.html, and ref 27). The compounds were sorted by hierarchical clustering using the algorithmic distance through the R package tool stats (https://www.r-project.org). The heatmap of the thermophoresis shift data was generated by the $\mathrm{R}$ package tool gplots. ${ }^{48}$

Compound Binding Sssays. Protein expression, microscale thermophoresis assays, and $K_{\mathrm{d}}$ measurements were done as described before. $^{20}$ The raw data of the thermophoresis assays can be found in Supporting Information, Table S4.

Pf Culture and L6 Toxicity Assays. Pf strain NF54 was cultured in human erythrocytes according to Trager and Jensen ${ }^{49}$ and protocols in www.mr4.org. The medium was RPMI 1640 supplemented with 0.5\% ALBUMAX II, $25 \mathrm{mM}$ Hepes, $25 \mathrm{mM} \mathrm{NaHCO}_{3}$ (pH 7.3), 0.36 $\mathrm{mM}$ hypoxanthine, and $100 \mu \mathrm{g} / \mathrm{mL}$ neomycin, and cultures were done at $37{ }^{\circ} \mathrm{C}$ in a humidified atmosphere of $3 \% \mathrm{O}_{2}, 4 \% \mathrm{CO}_{2}$, and $93 \% \mathrm{~N}_{2}$. $\mathrm{IC}_{50}$ values were determined in vitro by measuring the incorporation of the nucleic acid precursor $\left[{ }^{3} \mathrm{H}\right]$ hypoxanthine. ${ }^{50}$ Cytotoxicity was assessed with L6 cells, a primary cell line derived from rat skeletal myoblasts, as described elsewhere. ${ }^{51}$

\section{ASSOCIATED CONTENT}

\section{S Supporting Information}

The Supporting Information is available free of charge on the ACS Publications website at DOI: 10.1021/acs.jmedchem.6b00591.

Additional figure with binding data and tables with detailed docking data, comprehensive list of compounds with all of the measured parameters, complete data of microscale thermophoresis assays (PDF)

Precision docking (Glide, XP mode) of $5 \mathrm{E}$ and $5 \mathrm{~B}$ in PfHsp90 (XLSX)

Pose 1 of compound 5B (PDB)

Pose 2 of compound 5B (PDB)

Pose 1 of compound 5E (PDB)

SMILES molecular formula strings (CSV)

Detailed data of commercially obtained chemicals (ZIP)

Negative results of PAINS test (CSV)

\section{Accession Codes}

Authors will release the atomic coordinates and experimental data upon article publication.

\section{AUTHOR INFORMATION}

\section{Corresponding Author}

*Phone: +41 22379 6813. E-mail: didier.picard@unige.ch.

\section{Present Address}

"Tai Wang, Chemical Biology Program, Memorial Sloan Kettering Cancer Center, 417 East 68th Street, New York, NY 10065, USA.

\section{Author Contributions}

T.W. and D.P. designed the study and wrote the manuscript; T.W. performed most of the experiments; P.M. supervised and analyzed the $P f$ culture experiments; T.W. and D.P. analyzed all of the data.

\section{Notes}

The authors declare no competing financial interest.

\section{ACKNOWLEDGMENTS}

We are indebted to Sibylle Sax and Sonja Keller-Märki from the P.M. lab for technical assistance. The project was supported by grants from the Swiss National Science Foundation and by the Canton de Genève.

\section{ABBREVIATIONS USED}

CBZ, carbazole; Hsp90, heat-shock protein 90; MD, molecular dynamics; N-CBZ, aminoalcohol-carbazole; Pf, Plasmodium falciparum; SAR, structure-activity relationship

\section{REFERENCES}

(1) Hyde, J. E. Drug-resistant malaria - an insight. FEBS J. 2007, 274, 4688-4698.

(2) Ridley, R. G. Medical need, scientific opportunity and the drive for antimalarial drugs. Nature 2002, 415, 686-693.

(3) Flannery, E. L.; Chatterjee, A. K.; Winzeler, E. A. Antimalarial drug discovery - approaches and progress towards new medicines. Nat. Rev. Microbiol. 2013, 11, 849-862.

(4) Roy, K.; Ojha, P. K. Advances in quantitative structure-activity relationship models of antimalarials. Expert Opin. Drug Discovery 2010, $5,751-778$

(5) Picard, D. Preface to Hsp90. Biochim. Biophys. Acta, Mol. Cell Res. 2012, 1823, 605-606.

(6) Isaacs, J.; Whitesell, L. Hsp90 in Cancer: Beyond the Usual Suspects; Elsevier: New York, 2016; Vol. 129.

(7) Acharya, P.; Kumar, R.; Tatu, U. Chaperoning a cellular upheaval in malaria: heat shock proteins in Plasmodium falciparum. Mol. Biochem. Parasitol. 2007, 153, 85-94.

(8) Banumathy, G.; Singh, V.; Pavithra, S. R.; Tatu, U. Heat shock protein 90 function is essential for Plasmodium falciparum growth in human erythrocytes. J. Biol. Chem. 2003, 278, 18336-18345.

(9) Kumar, R.; Musiyenko, A.; Barik, S. The heat shock protein 90 of Plasmodium falciparum and antimalarial activity of its inhibitor, geldanamycin. Malar. J. 2003, 2, 30.

(10) Kumar, R.; Pavithra, S. R.; Tatu, U. Three-dimensional structure of heat shock protein 90 from Plasmodium falciparum: molecular modelling approach to rational drug design against malaria. J. Biosci. 2007, 32, 531-536.

(11) Pesce, E. R.; Cockburn, I. L.; Goble, J. L.; Stephens, L. L.; Blatch, G. L. Malaria heat shock proteins: drug targets that chaperone other drug targets. Infect. Disord.: Drug Targets 2010, 10, 147-157.

(12) Pallavi, R.; Roy, N.; Nageshan, R. K.; Talukdar, P.; Pavithra, S. R.; Reddy, R.; Venketesh, S.; Kumar, R.; Gupta, A. K.; Singh, R. K.; Yadav, S. C.; Tatu, U. Heat shock protein 90 as a drug target against protozoan infections: biochemical characterization of HSP90 from Plasmodium falciparum and Trypanosoma evansi and evaluation of its inhibitor as a candidate drug. J. Biol. Chem. 2010, 285, 37964-37975. 
(13) Shahinas, D.; Liang, M.; Datti, A.; Pillai, D. R. A repurposing strategy identifies novel synergistic inhibitors of Plasmodium falciparum heat shock protein 90. J. Med. Chem. 2010, 53, 3552-3557.

(14) Mout, R.; Xu, Z. D.; Wolf, A. K.; Davisson, V. J.; Jarori, G. K. Anti-malarial activity of geldanamycin derivatives in mice infected with Plasmodium yoelii. Malar. J. 2012, 11, 54.

(15) Shahinas, D.; Folefoc, A.; Pillai, D. R. Targeting Plasmodium falciparum Hsp90: Towards reversing antimalarial resistance. Pathogens 2013, 2, 33-54.

(16) Shahinas, D.; Folefoc, A.; Taldone, T.; Chiosis, G.; Crandall, I.; Pillai, D. R. A purine analog synergizes with chloroquine (CQ) by targeting Plasmodium falciparum Hsp90 (PfHsp90). PLoS One 2013, 8, e75446.

(17) Cowen, L. E.; Lindquist, S. Hsp90 potentiates the rapid evolution of new traits: drug resistance in diverse fungi. Science 2005, $309,2185-2189$.

(18) Cowen, L. E. The fungal Achilles' heel: targeting Hsp90 to cripple fungal pathogens. Curr. Opin. Microbiol. 2013, 16, 377-384.

(19) Dolgin, E.; Motluk, A. Heat shock and awe. Nat. Med. 2011, 17, 646-649.

(20) Wang, T.; Bisson, W. H.; Maser, P.; Scapozza, L.; Picard, D. Differences in conformational dynamics between Plasmodium falciparum and human Hsp90 orthologues enable the structure-based discovery of pathogen-selective inhibitors. J. Med. Chem. 2014, 57, $2524-2535$.

(21) Guiguemde, W. A.; Shelat, A. A.; Bouck, D.; Duffy, S.; Crowther, G. J.; Davis, P. H.; Smithson, D. C.; Connelly, M.; Clark, J.; Zhu, F.; Jimenez-Diaz, M. B.; Martinez, M. S.; Wilson, E. B.; Tripathi, A. K.; Gut, J.; Sharlow, E. R.; Bathurst, I.; El Mazouni, F.; Fowble, J. W.; Forquer, I.; McGinley, P. L.; Castro, S.; Angulo-Barturen, I.; Ferrer, S.; Rosenthal, P. J.; Derisi, J. L.; Sullivan, D. J.; Lazo, J. S.; Roos, D. S.; Riscoe, M. K.; Phillips, M. A.; Rathod, P. K.; Van Voorhis, W. C.; Avery, V. M.; Guy, R. K. Chemical genetics of Plasmodium falciparum. Nature 2010, 465, 311-315.

(22) Duffy, S.; Avery, V. M. Development and optimization of a novel 384-well anti-malarial imaging assay validated for highthroughput screening. Am. J. Trop. Med. Hyg. 2012, 86, 84-92.

(23) Derbyshire, E. R.; Prudencio, M.; Mota, M. M.; Clardy, J. Liverstage malaria parasites vulnerable to diverse chemical scaffolds. Proc. Natl. Acad. Sci. U. S. A. 2012, 109, 8511-8516.

(24) Meister, S.; Plouffe, D. M.; Kuhen, K. L.; Bonamy, G. M.; Wu, T.; Barnes, S. W.; Bopp, S. E.; Borboa, R.; Bright, A. T.; Che, J.; Cohen, S.; Dharia, N. V.; Gagaring, K.; Gettayacamin, M.; Gordon, P.; Groessl, T.; Kato, N.; Lee, M. C.; McNamara, C. W.; Fidock, D. A.; Nagle, A.; Nam, T. G.; Richmond, W.; Roland, J.; Rottmann, M.; Zhou, B.; Froissard, P.; Glynne, R. J.; Mazier, D.; Sattabongkot, J.; Schultz, P. G.; Tuntland, T.; Walker, J. R.; Zhou, Y.; Chatterjee, A.; Diagana, T. T.; Winzeler, E. A. Imaging of Plasmodium liver stages to drive next-generation antimalarial drug discovery. Science 2011, 334, 1372-1377.

(25) Gamo, F. J.; Sanz, L. M.; Vidal, J.; de Cozar, C.; Alvarez, E.; Lavandera, J. L.; Vanderwall, D. E.; Green, D. V.; Kumar, V.; Hasan, S.; Brown, J. R.; Peishoff, C. E.; Cardon, L. R.; Garcia-Bustos, J. F. Thousands of chemical starting points for antimalarial lead identification. Nature 2010, 465, 305-310.

(26) Nwaka, S.; Hudson, A. Innovative lead discovery strategies for tropical diseases. Nat. Rev. Drug Discovery 2006, 5, 941-955.

(27) Cao, Y.; Charisi, A.; Cheng, L. C.; Jiang, T.; Girke, T. ChemmineR: a compound mining framework for R. Bioinformatics 2008, 24, 1733-1734.

(28) Molette, J.; Routier, J.; Abla, N.; Besson, D.; Bombrun, A.; Brun, R.; Burt, H.; Georgi, K.; Kaiser, M.; Nwaka, S.; Muzerelle, M.; Scheer, A. Identification and optimization of an aminoalcohol-carbazole series with antimalarial properties. ACS Med. Chem. Lett. 2013, 4, 10371041.

(29) DeGraw, J. I.; Brown, V. H.; Keyanpour-Rad, M. 3- and 4carbazole dialkylaminocarbinols as potential antimalarial agents. J. Med. Chem. 1971, 14, 549-550.
(30) Thongthoom, T.; Songsiang, U.; Phaosiri, C.; Yenjai, C. Biological activity of chemical constituents from Clausena harmandiana. Arch. Pharmacal Res. 2010, 33, 675-680.

(31) Wang, T.; Echeverría, P. C.; Picard, D. Overview of molecular chaperones in health and disease. In Inhibitors of Molecular Chaperones as Therapeutic Agents; Machajewski, T. D., Gao, Z., Eds.; RSC Publishing: Cambridge, UK, 2014; p 1.

(32) Barta, T. E.; Veal, J. M.; Rice, J. W.; Partridge, J. M.; Fadden, R. P.; Ma, W.; Jenks, M.; Geng, L.; Hanson, G. J.; Huang, K. H.; Barabasz, A. F.; Foley, B. E.; Otto, J.; Hall, S. E. Discovery of benzamide tetrahydro-4H-carbazol-4-ones as novel small molecule inhibitors of Hsp90. Bioorg. Med. Chem. Lett. 2008, 18, 3517-3521.

(33) Nichols, S. E.; Baron, R.; Ivetac, A.; McCammon, J. A. Predictive power of molecular dynamics receptor structures in virtual screening. J. Chem. Inf. Model. 2011, 51, 1439-1446.

(34) Stebbins, C. E.; Russo, A. A.; Schneider, C.; Rosen, N.; Hartl, F. U.; Pavletich, N. P. Crystal structure of an Hsp90-geldanamycin complex: targeting of a protein chaperone by an antitumor agent. Cell 1997, 89, 239-250.

(35) Roe, S. M.; Prodromou, C.; O’Brien, R.; Ladbury, J. E.; Piper, P. W.; Pearl, L. H. Structural basis for inhibition of the Hsp90 molecular chaperone by the antitumor antibiotics radicicol and geldanamycin. $J$. Med. Chem. 1999, 42, 260-266.

(36) Immormino, R. M.; Kang, Y.; Chiosis, G.; Gewirth, D. T. Structural and quantum chemical studies of 8-aryl-sulfanyl adenine class Hsp90 inhibitors. J. Med. Chem. 2006, 49, 4953-4960.

(37) Corbett, K. D.; Berger, J. M. Structure of the ATP-binding domain of Plasmodium falciparum Hsp90. Proteins: Struct., Funct., Genet. 2010, 78, 2738-2744.

(38) Cumming, J. G.; Davis, A. M.; Muresan, S.; Haeberlein, M.; Chen, H. Chemical predictive modelling to improve compound quality. Nat. Rev. Drug Discovery 2013, 12, 948-962.

(39) Roy, K.; Kar, S.; Das, R. N. Understanding the Basics of QSAR for Applications in Pharmaceutical Sciences and Risk Assessment; Academic Press: New York, 2015.

(40) Irwin, J. J.; Shoichet, B. K. ZINC-a free database of commercially available compounds for virtual screening. J. Chem. Inf. Model. 2005, 45, 177-182.

(41) Pettersen, E. F.; Goddard, T. D.; Huang, C. C.; Couch, G. S.; Greenblatt, D. M.; Meng, E. C.; Ferrin, T. E. UCSF Chimera-a visualization system for exploratory research and analysis. J. Comput. Chem. 2004, 25, 1605-1612.

(42) Graves, A. P.; Shivakumar, D. M.; Boyce, S. E.; Jacobson, M. P.; Case, D. A.; Shoichet, B. K. Rescoring docking hit lists for model cavity sites: predictions and experimental testing. J. Mol. Biol. 2008, 377, 914-934.

(43) Friesner, R. A.; Murphy, R. B.; Repasky, M. P.; Frye, L. L.; Greenwood, J. R.; Halgren, T. A.; Sanschagrin, P. C.; Mainz, D. T. Extra precision glide: docking and scoring incorporating a model of hydrophobic enclosure for protein-ligand complexes. J. Med. Chem. 2006, 49, 6177-6196.

(44) Halgren, T. A.; Murphy, R. B.; Friesner, R. A.; Beard, H. S.; Frye, L. L.; Pollard, W. T.; Banks, J. L. Glide: a new approach for rapid, accurate docking and scoring. 2. Enrichment factors in database screening. J. Med. Chem. 2004, 47, 1750-1759.

(45) Friesner, R. A.; Banks, J. L.; Murphy, R. B.; Halgren, T. A.; Klicic, J. J.; Mainz, D. T.; Repasky, M. P.; Knoll, E. H.; Shelley, M.; Perry, J. K.; Shaw, D. E.; Francis, P.; Shenkin, P. S. Glide: a new approach for rapid, accurate docking and scoring. 1. Method and assessment of docking accuracy. J. Med. Chem. 2004, 47, 1739-1749.

(46) Case, D. A.; Cheatham, T. E., 3rd; Darden, T.; Gohlke, H.; Luo, R.; Merz, K. M., Jr.; Onufriev, A.; Simmerling, C.; Wang, B.; Woods, R. J. The Amber biomolecular simulation programs. J. Comput. Chem. 2005, 26, 1668-1688.

(47) Harvey, M. J.; Giupponi, G.; Fabritiis, G. D. ACEMD: Accelerating biomolecular dynamics in the microsecond time scale. J. Chem. Theory Comput. 2009, 5, 1632-1639.

(48) Warnes, G. R.; Bolker, B.; Bonebakker, L.; Gentleman, R.; Huber, W.; Liaw, A.; Lumley, T.; Maechler, M.; Magnusson, A.; 
Moeller, S. gplots: Various R Programming Tools for Plotting Data, R package version 2, 2009.

(49) Trager, W.; Jensen, J. B. Human malaria parasites in continuous culture. 1976. J. Parasitol. 2005, 91, 484-486.

(50) Desjardins, R. E.; Canfield, C. J.; Haynes, J. D.; Chulay, J. D. Quantitative assessment of antimalarial activity in vitro by a semiautomated microdilution technique. Antimicrob. Agents Chemother. 1979, 16, 710-718.

(51) Regalado, E. L.; Tasdemir, D.; Kaiser, M.; Cachet, N.; Amade, P.; Thomas, O. P. Antiprotozoal steroidal saponins from the marine sponge Pandaros acanthifolium. J. Nat. Prod. 2010, 73, 1404-1410. 